IMAGE IN

CARDIOLOGY

\title{
An unusual cause for a dilated right heart 33-years post-surgical repair of aortic coarctation
}

\author{
Charles G. Kyriakakis", Annari van Rensburg*, \\ Ntobeko A.B. Ntusi", Jacques T. Janson', Philip G. Herbst* \\ and Anton F. Doubell"
}

"Division of Cardiology, Department of Medicine, Stellenbosch University and Tygerberg Hospital, Cape Town, South Africa "Department of Medicine, University of Cape Town and Groote Schuur Hospital, Cape Town, South Africa

Division of Cardiothoracic Surgery, Department of Surgery, Stellenbosch University and Tygerberg Hospital, Cape Town, South Africa

Address for correspondence:

Dr Charles G. Kyriakakis

Division of Cardiology

Tygerberg Academic Hospital

PO Box 19063

Tygerberg

7505

South Africa

Email:

cgk@sun.ac.za

\section{INTRODUCTION}

Prior to the surgical correction of a congenital cardiac defect in childhood, an in-depth evaluation for potential co-existing anomalies is mandatory. Haemodynamic sequelae consequent to a previously unidentified associated defect may only become apparent in adulthood, necessitating a clinical review and detailed search for their cause.

\section{CASE STUDY}

A 40-year-old female with Turner's syndrome and a prior surgical aortic coarctation repair at the age of 7 was referred to our grown-up congenital heart disease clinic for evaluation of a cardiac murmur. Other than occasional palpitations she had remained asymptomatic. Features of neither right nor left heart failure could be found on clinical examination and upper limb blood pressures were normal, without radio-femoral delay. The apex beat was found to be lateral to the mid-clavicular line in the presence of a parasternal heave and a fixed split second heart sound with a 2/6 systolic murmur at the pulmonary area.

\section{ABSTRACT}

Prior to planning for the surgical correction of a congenital cardiac defect it is of the utmost importance that additional defects, which themselves might also require surgical correction, be sought and identified. Of these, those leading to volume overload of the right heart, and particularly those that are not easily identified on transthoracic echocardiography, may go unnoticed during initial evaluation in childhood. We describe the approach to such a clinical problem, highlighting the value of multimodality imaging in this context, and outline the options available for surgical correction. SAHeart 20।8;15:220-224

The left ventricle was non-dilated with preserved systolic function on transthoracic echocardiography (TTE). The right atrium (RA) and right ventricle (RV) were dilated with D-shaping of the interventricular septum during diastole, in keeping with a volume loading condition of the right heart. There was no evidence of either severe tricuspid or pulmonary regurgitation, and colour Doppler examination of the interatrial septum suggested it was intact. Pulmonary artery systolic pressure was estimated to be $36 \mathrm{mmHg}$. The aortic valve was not bicuspid and no recurrence of coarctation was found. A dilated coronary sinus (CS) of $27 \mathrm{~mm}$ was evident in the apical 2-chamber view (Figure I), alerting the echocardiographer to the possibility of a persistent left sided vena cava (PLSVC). Agitated saline contrast injected via the left antecubital vein opacified the CS first, followed by direct spillage of contrast into the left atrium (LA) and then left ventricle (Video I). This confirmed the presence of a PLSVC and unroofed CS as the cause of a left-to-right shunt at atrial level. A PLSVC draining to an intact CS would not have any haemodynamic effect on the right heart, as venous return entering the PLSVC would be directed into the RA via the intact CS. However, when an unroofed CS is present the result is left-to-right shunting at atrial level, this being the rarest form of atrial septal defect (ASD). During a single R-R cycle on TTE the CS appeared to be completely unroofed (Figure 2).

Surgical correction was planned due to the haemodynamic consequence of right heart dilatation secondary to this pre- 

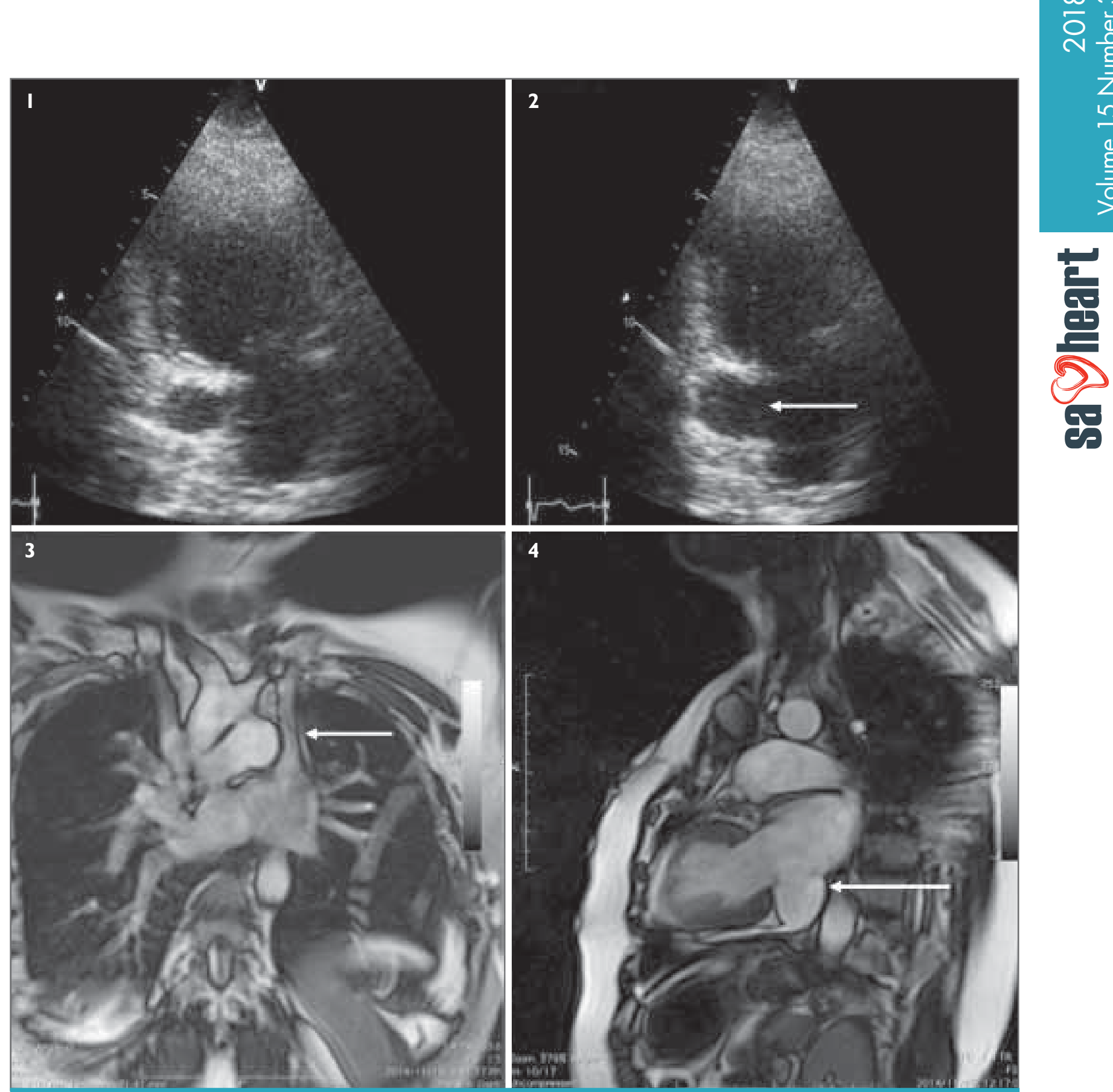

FIGURE I: TTE apical 2-chamber view, demonstrating a dilated CS.

FIGURE 2: During a single R-R cycle the CS appears to be unroofed.

FIGURE 3: Coronal MRI view. Both the right-sided SVC and the PLSVC are visualised, entering the roof of the RA and LA, respectively.

FIGURE 4: Horizontal long axis (2-chamber) view on sagittal MRI. The CS is demonstrated to be enlarged and completely unroofed.

viously undiagnosed defect. A transoesophageal echocardiogram (TOE) provided confirmation of the PLSVC and unroofed CS during a repeated agitated saline injection via the left arm (Video 2). It also allowed for all 4 pulmonary veins to be identified draining into the LA, excluding the possible association of anomalous pulmonary venous drainage. A shunt fraction of 2.5:I and pulmonary vascular resistance of 1.8 Wood units was found at left and right heart study, confirming suitability for surgical correction. Injection of iodinated contrast via the left forearm during this study allowed for the identification of a possible bridging vein connecting the PLSVC to the right-sided SVC. This was followed by contrast extravasation into the LA via the PLSVC and unroofed CS (Video 3). The anatomy and calibre of this bridging vein was defined via cardiovascular magnetic resonance (CMR), allowing for more accurate surgical planning. CMR also revealed that the PLSVC entered the roof of the LA between the left atrial appendage and the left upper pulmonary vein, separate to the completely unroofed CS (Figures 3 and 4), which is a Type I Kirklin and Barrat-Boyes coronary sinus defect, also known as Raghib's syndrome.(1) 


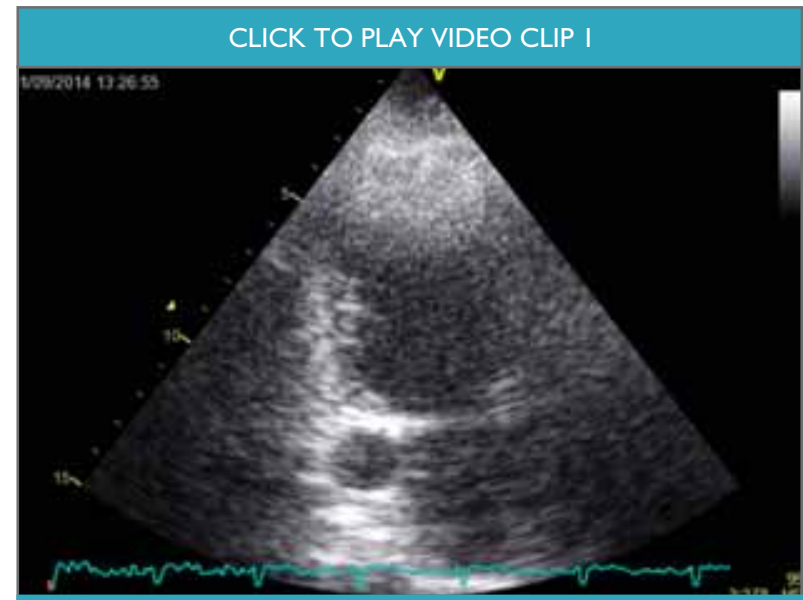

VIDEO I: Apical 2-chamber TTE view during agitated saline contrast injection via the left cubital vein. Contrast is seen to enter the CS first followed by direct spillage into the LA, confirming the CS to be unroofed.

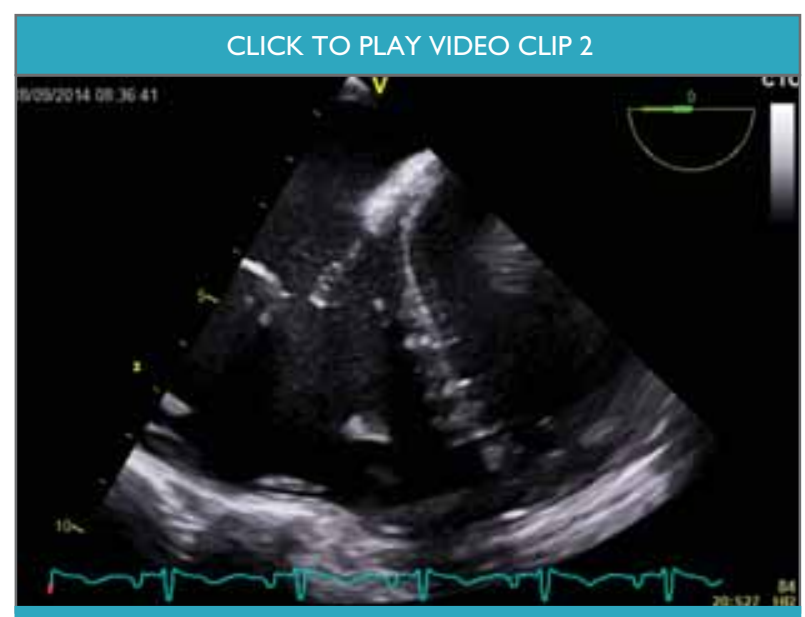

VIDEO 2: Low oesophageal 4-chamber TOE view at the level of the CS during agitated saline contrast injection from the left cubital vein. A defect is apparent in the postero-inferior portion of the interatrial septum and the roof of the CS is absent. Saline contrast enters the LA superiorly via the PLSVC and then shunts into the RA across the CS-ASD. Note the dilated right ventricle.

At surgery the CS in our patient was confirmed to be completely unroofed with an associated coronary sinus ASD (Video 4). In the setting of a PLSVC with completely unroofed CS, a CS associated-ASD is present in the postero-inferior region of the interatrial septum in the usual position of the coronary sinus ostium. ${ }^{(2)}$ Whilst this posteriorly positioned defect in the interatrial septum could not be visualised on TTE, it was evident on both TOE and CMR. Surgical correction included ligation of the PLSVC, which entered the LA superiorly, and patching of the CS-ASD with a pericardial patch. The unroofed portion of the CS within the LA was not baffled, leaving a clinically non-significant intracardiac right-toleft shunt at atrial level.
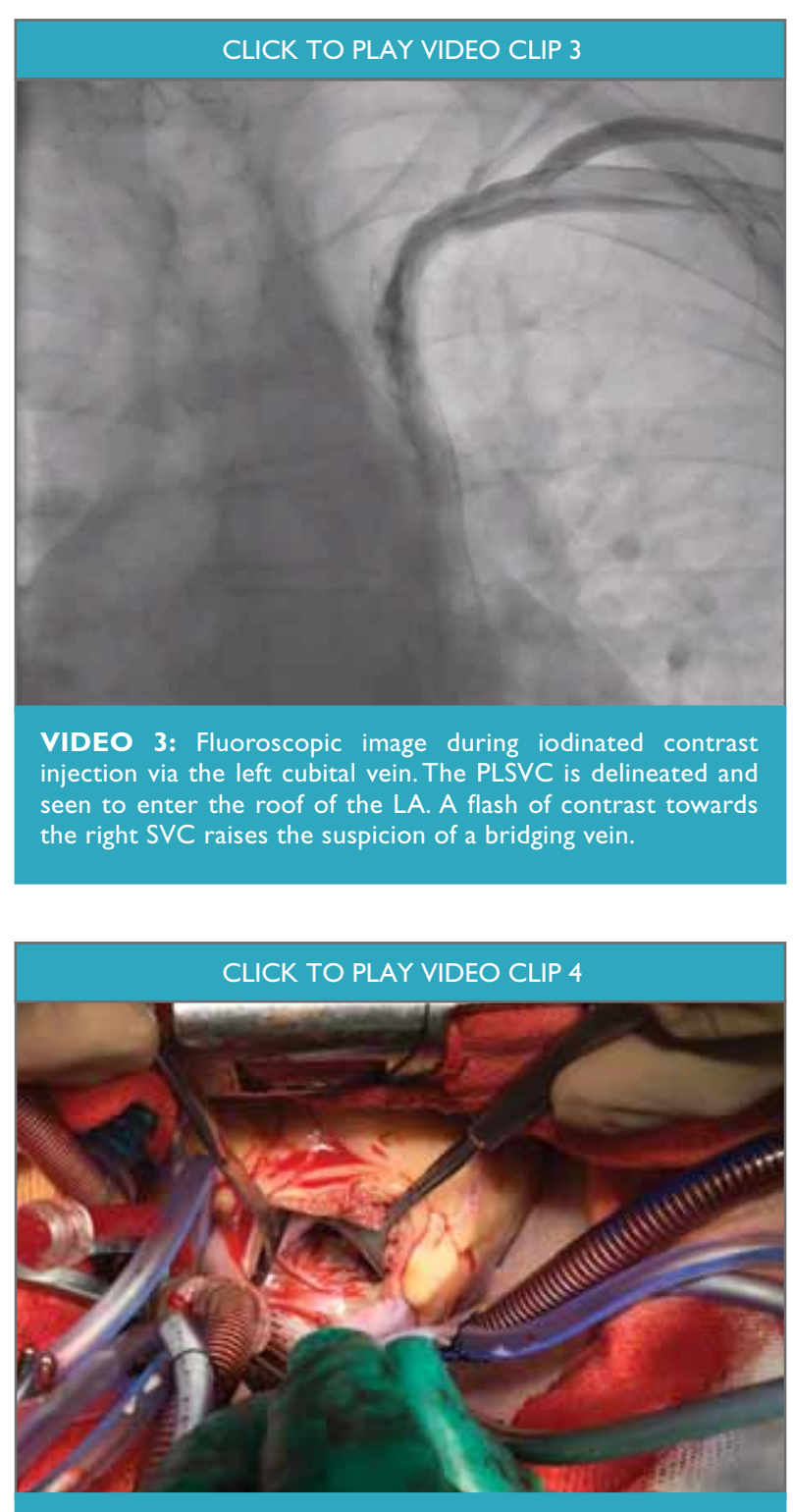

VIDEO 4: Intra-operatively, via right atriotomy, the anatomy of the interatrial septum is delineated. The surgeon first points to the tricuspid valve followed by the large CS-ASD, and finally the intact secundum septum.

\section{DISCUSSION}

PLSVC is the most common congenital venous malformation and is often an incidental finding at pacemaker implantation, central venous catheterisation or TTE for an unrelated indication. It is present in $5 \%-10 \%$ of patients with other congenital cardiac lesions and in 13\% of patients with Turner's syndrome. ${ }^{(3)}$ Although the association of a PLSVC with unroofed CS is rare, this association commonly remains undetected at the time of surgical correction of an associated congenital cardiac defect in childhood. ${ }^{(4)}$

TTE does not provide accurate delineation of posteriorly situated cardiac structures such as the CS, whilst TOE and 
CMR offer a more precise anatomical evaluation of deepseated cardiac structures. ${ }^{(5)}$ During TTE examination the CS is best visualised in its short axis in an apical 2-chamber view, and in its long axis in an apical 4-chamber view with posterior tilt of the imaging plane towards the left atrioventricular (AV) groove. Dilatation of the CS in the presence of volume overload of the right heart is suggestive of an unroofed CS defect when an ASD cannot be identified. ${ }^{(4)}$ At TOE the CS can be well visualised by slowly advancing the probe from a midoesophageal 4-chamber view, keeping the LA centred in the imaging plane, until it is positioned just posterior to the left A-V groove. This allows for the full length of the CS to be clearly delineated as it enters the RA via its ostium. Unroofing can be complete or partial, being limited to either the mid or alternatively the distal portion of the CS.(I) 2-D evaluation provides for the assessment of potential defects along the roof of the CS and optimised colour Doppler mapping, with a slightly reduced scale of $35-40 \mathrm{~cm} / \mathrm{sec}$, will allow for the confirmation of a potential CS defect.(6) At TOE the inferoposterior aspect of the IAS can also be assessed for a possible CS-associated ASD in the region of the CS ostium. A useful view to evaluate for this is achieved by advancing the multiplane angle from a standard bicaval view $\left(105^{\circ}\right)$, to between $110^{\circ}-120^{\circ}$ with slight advancement of the probe allowing for assessment of the inferior portion of the IAS and CS ostium. ${ }^{(6)}$ PLSVC can be associated with a CS defect and its presence can be confirmed at TOE by agitated saline bubble contrast injection from the left cubital vein. ${ }^{(1,6)}$

Anomalous pulmonary venous drainage and sinus venosus defects are 2 further uncommon ASD equivalents that can be challenging to diagnose via TTE due to their posterior position within the cardiac mass. ${ }^{(6)}$ It is frequently possible to delineate all 4 pulmonary veins (PVs) on a TTE apical 4-chamber view, with slight anterior tilt required to identify the right upper pulmonary vein (RUPV). An alternative view for assessing whether or not all 4 PVs enter the LA is the so called "crab view" of the LA acquired from the suprasternal window. In Scimitar syndrome, anomalous drainage of the right lung via the Scimitar vein to the IVC can be demonstrated on a subcostal bicaval view, similarly it is also possible to identify an anomalous RUPV entering the SVC using the same view.

In total anomalous pulmonary venous return (TAPVR) all 4 PV's drain to the right heart via a common vein, or alternatively directly to the RA. (7) To be compatible with life, rightto-left intracardiac shunting must occur via either an ASD or patent foramen ovale, or more rarely a PDA. Pulmonary venous drainage can either be extracardiac via a vertical vein to either the SVC or IVC, or alternativley intracardiac directly to the coronary sinus. (7) In the supracardiac form of TAPVR all 4 pulmonary veins unite behind the heart and drain to the left innominate vein via a vertical vein. ${ }^{(7)}$ This vertical vein courses superiorly towards the left innominate vein and the suprasternal TTE view is ideal for identifying this. In a standard suprasternal imaging plane used to assess the aortic arch, colour Doppler mapping will identify flow towards the probe via the vertical vein, and not away from it as would be expected, alerting the sonographer to a potential diagnosis. Similarly, in infracardiac TAPVR, a vertical vein directs pulmonary venous bloodflow to either the suprahepatic segment of the IVC, located above the diaphragm, or alternatively below the diaphragm to the hepatic veins. A subcostal view assessing the IVC will be useful here. The finding of a dilated CS, with continuous flow within it on colour Doppler imaging, in the presence of an ASD, would raise the suspicion of the intracardiac variant of TAPVR. Whilst patients with either the supra- or intracardiac variants of TAPVR may first come to medical attention in adolescence or early adulthood, those with the infracardiac variant commonly present in the neonatal period with pulmonary oedema due to obstruction along the course of their pulmonary venous return. ${ }^{(7)}$

In the setting of a volume loaded and dilated RV the identification of each individual PV on TTE can however become difficult. TOE more accurately allows for the delineation of pulmonary venous drainage compared to TTE, whilst CMR provides for a more accurate outline of the precise anatomical course of each individual PV when anomalous PV drainage is being considered. ${ }^{(7)}$

Identification of both superior and inferior sinus venosus defects is also possible via TTE with a reported sensitivity of $12 \%-44 \%{ }^{\left({ }^{8}\right)}$ The most useful view providing for this is a modification of the subcostal midatrial view..$^{(9)}$ Slight clockwise rotation with anterosuperior tilt of the probe allows for the visualisation of the superior aspect of the IAS at the entrance of the SVC. Superior sinus venosus defects can be identified using this technique. (9) An alternative approach is to acquire a subcostal bicaval view and slowly rotate anticlockwise to approximately a 2 o'clock position. In this view, the RA is in the near field and the LA in the far field of the image, with the IVC on the left-hand side and the SVC on the right. This view also allows for detailed evaluation of the superior portion of the IAS and provides the added advantage of including the RUPV in the scan sector. Superior sinus venosus defects are commonly associated with anomalous PV drainage of the RUPV to the RA. ${ }^{\left({ }^{6}\right)}$ In the presence of inadequate subcostal 
windows, a high right parasternal view can be valuable for diagnosing superior sinus venosus defects. ${ }^{(6)}$ This view is obtained with the patient in the right lateral decubitus position with the probe in a superior-inferior orientation. It provides perpendicular alignment of the IAS to the ultrasound beam with the SVC visualised in its long axis. ${ }^{(6)}$ Inferior sinus venosus defects can be delineated by scrutinising the IVC and RA junction in a modified subcostal bicaval view that allows for inclusion of the LA in the scan plane. However, due to their posterior position, TOE is a more accurate means by which to identify either of these rare defects. ${ }^{(6)}$ The mid-oesophageal bicaval view, commonly acquired at $105^{\circ}-110^{\circ}$, delineates the junction of both the SVC and IVC with the RA, allowing for the identification of defects at either of these positions. ${ }^{(6)}$ Sinus venosus defects are frequently small in size on 2-D imaging, inadequately accounting for RV volume loading on their own. It is therefore mandatory to search for associated anomalous PV drainage, particularly of the RUPV in a superior sinus venosus defect. ${ }^{(6)}$ The RUPV can be visualised using a mid-oesophageal bicaval view, and anomalous drainage to the RA can be identified here. ${ }^{(6)}$ A pathognomonic anatomical aspect of sinus venosus defects is straddling of either the SVC in the setting of a superior sinus venosus defect, or alternatively the IVC in the setting of an inferior defect, over the IAS.(10) This should be sought for during TOE examination. Whilst TTE and TOE are the firstline modalities for the evaluation of shunt lesions at atrial level, advanced imaging modalities, particularly CMR, are valuable in assessing those that are secondary to posteriorly positioned defects. ${ }^{(5)}$

The consequences of not addressing an unroofed CS at the time of original surgical intervention for another defect are those of the haemodynamic effects of a left-to-right shunt at atrial level, namely right heart dilatation and failure, atrial tachyarrhythmias, and the potential for paradoxical thromboembolism and brain abscess. ${ }^{(2)}$ The presence of a bridging vein from a PLSVC to right SVC is of surgical relevance; if there is one present the PLSVC can be ligated distally leaving correction of the unroofed CS as the main surgical intervention. A potential surgical approach is to not address the unroofed CS and rather only patch the associated CS-ASD, as was performed in our patient, leaving a negligible $(<5 \%)$ right-to-left intra-cardiac shunt, i.e. coronary venous to LA. (I) Should a bridging vein not be present, complex baffling of the PLSVC through the LA to redirect blood flow to the RA would need to be undertaken. ${ }^{(1)}$

Alternative surgical strategies could include either: redirecting the PLSVC to the left pulmonary artery as a bidirectional superior cavopulmonary shunt and patching the CS-ASD, or alternatively, as has been more recently described, anastamosing the PLSVC to the right atrial appendage with patch closure of the CS-ASD.(I) Extracardiac corrective procedures, i.e. that exclude the need for complex intracardiac baffling to redirect blood flow, reduce the risk for future atrial arrhythmia and also the possible development of obstruction to pulmonary venous blood flow.

\section{CONCLUSION}

ASD equivalents, including unroofed coronary sinus defects, anomalous pulmonary venous drainage and sinus venosus defects, are notoriously challenging to diagnose on TTE due to their posterior position. TOE and CMR offer a more detailed evaluation of posteriorly seated cardiac structures compared to TTE. CMR also provides for a more comprehensive assessment of the defect and assists with surgical planning prior to the correction thereof.

\section{Conflict of interest: none declared.}

\section{REFERENCES}

I. Kouchoukos NT, Blackstone EH, Hanley FL, et al. Unroofed coronary sinus syndrome. Kirklin/Barratt-Boyes - Cardiac Surgery. 4th ed. Elsevier Saunders; 2013:1217-1226.

2. Quaegebeur J, Kirklin JW, Pacifico AD, et al. Surgical experience with unroofed coronary sinus. Ann of Thorac Surg 1979;27:418.

3. Ho VB, Bakalov VK, Cooley M, et al. Major vascular anomalies in Turner Syndrome: Prevalence and magnetic resonance angiographic features. Circulation 2004; 1 10:1694- 1700.

4. Pérez Matos AJ, Planken RN, Bouma BJ, et al. Unroofed coronary sinus newly diagnosed in adult patients after corrected congenital disease. Neth Heart J 2014;22:240-245.

5. Hahm JK, Park YW, Lee JK, et al. Magnetic resonance imaging of unroofed coronary sinus: Three cases. Pediatric Cardiology 2000;2I (4):382e7.

6. Silvestry FE, Cohen MS, Armsby LB, et al. Guidelines for the echocardiographic assessment of atrial septal defect and patent foramen ovale: From the American Society of Echocardiography and Society for Cardiac Angiography and Interventions. J Am Soc Echocardiogr 2015;28:9 10-958.

7. Katre R, Burns SK, Murillo H, et al. Anomalous pulmonary venous connections. Semin Ultrasound CT MRI 2012;33:485-499.

8. Shub C, Dimopoulos IN, Seward JB, et al. Sensitivity of two-dimensional echocardiography in the direct visualisation of atrial septal defect utilising the subcostal approach: Experience with 154 patients. J Am Coll Cardiol 1983;2(1): 127-135.

9. Nasser FN, Tajik AJ, Hagler DJ. Diagnosis of sinus venosus atrial septal defect by two-dimensional echocardiography. Mayo Clin Proc 1981;56:568-572.

10. Oliver JM, Gallego P, Gonzalez A, et al. Sinus venosus syndrome: Atrial septal defect or anomalous venous connection? A multiplane transoesophageal approach. Heart 2002;88:634-638.

I I. Pérez-Caballero R, Izquierdo BP, Gil-Jaurena JM. Raghib Syndrome. Surgical treatment. Rev Esp Cardiol 2016;69(1):71. 